\title{
Efeito da Aplicação de Thiamethoxam para o Controle do Tripes na Redução da Severidade da Verrugose do Amendoim*
}

\author{
Andrea R. A. Moraes ${ }^{1}$, Sérgio A. Moraes ${ }^{1}$, André L. Lourenção ${ }^{1}$, Ignácio J. Godoy² \\ \& Antônio Lúcio M. Martins ${ }^{3 * *}$ \\ ${ }^{1}$ Centro de Fitossanidade, IAC; ${ }^{2}$ Centro APTA de Grãos e Fibras, IAC, Cx. Postal 28, CEP 13001-970, \\ Campinas, SP, e-mail: sergioam@iac.sp.gov.br; ${ }^{3}$ Pólo Regional de Desenvolvimento Centro-Norte, \\ APTA, IAC, CEP 15830-000, Pindorama, SP
}

(Aceito para publicação em 20/03/2006)

Autor para correspondência: Sérgio Almeida de Moraes

MORAES, A.R.A., MORAES, S.A., LOURENÇÃO, A.L., GODOY, I.J. \& MARTINS, A.L.M. Efeito da aplicação de thiamethoxam para controle do tripes na redução da severidade da verrugose do amendoim. Fitopatologia Brasileira 31:164170. 2006.

\section{RESUMO}

A relação entre a aplicação do thiamethoxam para o controle do tripes e a severidade da verrugose foi verificada em experimentos instalados em Campinas e Pindorama, em 2001/2002, no esquema de parcelas subdivididas. As parcelas corresponderam aos tratamentos, com e sem controle do tripes com thiamethoxam e as subparcelas aos seis cultivares de amendoim, IAC-Tatu-ST, IAC-5, IAC-22 (eretos, de ciclo curto), Runner IAC-886, Tégua e IAC-Caiapó (rasteiros, de ciclo longo). Foram utilizadas as avaliações de número de tripes por folíolo e nota de sintomas obtidos aos 56 e 57 dias do plantio, em Campinas e Pindorama, quando foram registradas as maiores populações do inseto nesses locais. A severidade da verrugose foi avaliada aos 79 e 91 dias após o plantio, em Campinas e Pindorama, respectivamente, através de escala de notas baseada nos sintomas exibidos nas hastes e pecíolos. Os resultados mostraram a eficiência do inseticida thiamethoxam no controle do tripes em todos os cultivares. Nos dois locais observou-se a redução na severidade da verrugose, quando foi aplicado o thiamethoxam. A severidade da verrugose foi maior nos cultivares de porte ereto, com o IAC-Caiapó apresentando os menores índices de severidade. A produção foi maior nas parcelas tratadas com o inseticida, nos dois locais, tendo o IACCaiapó apresentado produções superiores aos demais cultivares. A ação do thiamethoxam controlando o tripes e reduzindo a severidade da verrugose deve ser melhor explorada nas práticas de manejo integrado.

Palavras-chave adicionais: Sphaceloma arachidis, Enneothrips flavens, inseticida.

\begin{abstract}
Effect of thiamethoxam application to thrips control on the reduction of peanut scab severity

The relation among the application of thiamethoxam for thrips control and the severity of peanut scab was evaluated in field experiments runned at Campinas and Pindorama, during 2001/2002, in split-plot design. Main plots consisted of the control and no control of thrips with thiamethoxam and, subplots, of six peanut cultivars: IAC-Tatu-ST, IAC-5, IAC-22 (upright type, short cycle), Runner IAC-886, Tegua and IAC-Caiapo (runner type, long cycle). The evaluations of number of thrips per leaflet and ratingof symptoms, were performed at 56 and 57 days after sowing date in Campinas and Pindorama, when the highest insect populations where registered. In both experiments a partial control of peanut scab was observed, and so, its severity was evaluated at 79 and 91 days of the plantation, in Campinas and Pindorama, respectively, using a rating scale based on symptons shown in stems and petioles. The results showed the efficiency of thiamethoxam in controling thrips in all cultivars. In both localities was observed a reduction in the severity of peanut scab, when thiamethoxam was applied. The severity of scab was higher in the upright cultivars, with the IAC-Caiapo showing the lower scores of severity. The yield was higher for the parcels treated with the insecticide, in both localities, and IAC-Caiapo presented superior yields in relation to the other cultivars. The action of thiamethoxam controling thrips and reducing scab severity should be more explored in integrated control practices.
\end{abstract}

Additional keywords: Sphaceloma archidis, Enneothrips flavens, insecticide.

\section{INTRODUÇÃO}

A cultura do amendoim (Arachis hypogaea L.) é normalmente afetada por várias pragas e doenças e, com

\footnotetext{
*Parte da Dissertação de Mestrado do primeiro autor. Instituto Agronômico de Campinas. 2005.
}

freqüência, os prejuízos são consideráveis se o controle fitossanitário não for realizado, ou se for conduzido precariamente (Lasca et al., 1983; Moraes \& Godoy, 1997). Entre as pragas, os tripes Enneothrips flavens e Caliothrips brasiliensis (Thysanoptera: Thripidae) são considerados os mais importantes, pelos prejuízos causados, ocorrência generalizada nas culturas e elevados níveis 
populacionais (Calcagnolo et al., 1974; Gallo et al., 2002).

No Estado de São Paulo, o controle mais eficiente do tripes tem sido através da utilização de inseticidas, onde normalmente são feitas de três a seis pulverizações durante o ciclo da cultura (Lasca et al., 1983).

Entre os inseticidas mais recentes e estudados disponíveis no mercado, o thiamethoxam tem sido testado com sucesso em muitas espécies vegetais de importância econômica no controle de insetos sugadores (Benvenga et al., 1998; Raetano et al., 2000; Martins \& Nakamura, 2000; Moraes et al., 2005; Senn et al., 2000a, b). A molécula que o compõe pertence à classe química dos neonicotinóides, que interferem com o receptor de acetilcolina dos insetos (Senn et al., 2000b). Esse produto, quando usado no tratamento de sementes, controlou $90 \%$ de pulgões e tripes do algodoeiro nas doses de 210 e $300 \mathrm{~g} / 100 \mathrm{~kg}$ de sementes (Belletini et al., 2000). Hofer et al. (2000) relataram que o produto promove o desenvolvimento inicial das plantas devido ao seu eficiente controle das pragas.

A cultura é mais sensível ao ataque da praga desde a germinação até 50-70 dias de idade, sendo que o período crítico de ataque situa-se entre os 50 e 60 dias após a germinação da planta (Batista et al., 1973; Gabriel et al., 1996 e 1998; Moraes et al., 2005).

Gabriel et al. (1996, 1998), estudando a flutuação populacional do tripes E. flavens em sete cultivares de amendoim, encontraram diferenças entre os cultivares, tendo os cultivares de hábito de crescimento rasteiro (grupo Virgínia) aparentemente mostrado menor número de insetos. Nesse estudo, os autores observaram que os cultivares de ciclo longo IAC-Caiapó e IAC-Jumbo tenderam a serem menos atacados pelo tripes na ausência de controle químico, enquanto que os precoces, como 'Tatu', foram mais atacados.

Entre as doenças da cultura do amendoim, a verrugose, causada por Sphaceloma arachidis Bitanc. \& Jenkins, constatada pela primeira vez por Bitancourt \& Jenkins (1940), pode ser considerada uma doença importante para as condições de cultivo do Estado de São Paulo quando ocorre nos estágios iniciais de crescimento da cultura, prejudicando o desenvolvimento de plantas severamente afetadas (Moraes \& Godoy, 1997).

Entre as medidas de controle desta doença, a rotação de culturas e o controle químico são as mais recomendadas (Barreto, 1997; Kokalis-Burelle et al., 1997; Moraes \& Godoy, 1997). A utilização de cultivares resistentes é outra forma de controle da verrugose (Barreto, 1997; Moraes \& Godoy, 1997; Moraes et al., 2001).

A cultivar Tatu, ainda plantada, é suscetível à verrugose, sendo necessário o uso de fungicidas para o seu controle (Moraes \& Godoy, 1997). A utilização de fungicidas do grupo dos triazóis, principalmente o difenoconazole, para o controle das manchas castanha e preta tem proporcionado bom controle da verrugose (Barreto, 1997; Dario et al., 1994; Leite et al., 1994; Moraes et al., 2001).

Rossetto et al. (1968) relataram a diminuição da severidade da verrugose quando o tripes foi controlado por inseticidas à base de demeton-S-metil (Metasystox). No entanto, os detalhes desta interação ainda não foram bem esclarecidos.

Os experimentos aqui relatados foram inicialmente conduzidos visando estudar a incidência natural de tripes em seis cultivares, comparando o seu desempenho, com e sem controle da praga (Moraes, 2005). Porém, como se verificou a incidência da verrugose nos dois experimentos procurouse verificar a interação entre a aplicação do thiamethoxam para o controle do tripes e a severidade da verrugose.

\section{MATERIAL E MÉTODOS}

Foram conduzidos experimentos em condições de campo, no Centro Experimental do Instituto Agronômico de Campinas, SP e no Pólo Regional de Desenvolvimento Centro-Norte, Unidade Experimental de Pindorama, SP.

De acordo com Moraes (2005), os experimentos foram instalados em 10 e 11/12/2001, em Campinas e Pindorama, respectivamente, em esquema de parcelas subdivididas, com delineamento em blocos ao acaso, com seis repetições. As parcelas corresponderam aos tratamentos, com e sem controle químico do tripes (parcelas tratadas e parcelas não tratadas) e, as subparcelas, as seis cultivares de amendoim, sendo três deles de hábito de crescimento ereto e ciclo curto, IAC-Tatu-ST, IAC-5, IAC-22, e três de hábito de crescimento rasteiro e ciclo longo, Runner IAC 886, Tégua e IAC-Caiapó (Godoy et al., 1996, 2001; IAC, 2000, 2003).

Nas parcelas com controle da praga, as sementes foram tratadas antes do plantio com thiamethoxam (na formulação $700 \mathrm{WS}$ do produto comercial), na dose de $100 \mathrm{~g}$ do produto/ $100 \mathrm{~kg}$ de sementes. A partir dos 40 dias após o plantio, as parcelas foram pulverizadas com thiamethoxam, na formulação de granulado dispersível, (na formulação 250 WG do produto comercial), na dosagem de $100 \mathrm{~g} / \mathrm{ha}$, sendo estas pulverizações repetidas a cada 15 dias, num total de cinco aplicações, até os 30 dias antes da colheita das cultivares.

Cada subparcela constou de quatro linhas de $10 \mathrm{~m}$ de comprimento de cada cultivar. O espaçamento entre linhas utilizado foi o recomendado de acordo com o tipo vegetativo da cultivar, ou seja, de $90 \mathrm{~cm}$ para as cultivares rasteiras e de $60 \mathrm{~cm}$ para os de porte ereto.

As doenças foliares foram controladas em todas as parcelas, através de pulverizações com fungicida sistêmico à base de difenoconazole, na dosagem de $0,35 \mathrm{~L} \mathrm{ha}^{-1}$, iniciandose as pulverizações aos 50 dias do plantio, e prosseguindo a cada 15 dias, até próximo ao final do ciclo de cada cultivar, num total de cinco aplicações.

Em ambas as localidades, as contagens de tripes foram realizadas aos 56 e 57 dias do plantio (DAP). Em cada data, foram coletados dez brotos apicais por subparcela, conforme metodologia descrita por Moraes (2005). Além disso, foram coletadas amostras de dez folíolos desenvolvidos e semi-abertos (apenas um de cada folha), ao acaso, de 
cada subparcela. Esses folíolos foram coletados de folhas maduras, da parte do meio da planta, sendo atribuída a cada folíolo uma nota de 1 a 5 , segundo uma escala visual de acordo com a intensidade de sintomas de prateamento nas folhas (Moraes, 2005).

A avaliação da severidade da verrugose deu-se aos 79 e 91 DAP, em Campinas e Pindorama, respectivamente, através de escala de notas de 1 a 4, baseada nos sintomas exibidos nas hastes e pecíolos de dez plantas por parcela, de acordo com a metodologia citada por Moraes et al. (2001).

A nota média dos sintomas e o índice de doença (variando de 0 a $100 \%$ ) foram obtidos da seguinte forma:

Nota média $=n 1 * 1+n 2 * 2+n 3 * 3+n 4 * 4) / N$ :

Índice de doença $(\%)=(\mathrm{n} 1 * 0+\mathrm{n} 2 * 25+\mathrm{n} 3 * 50+$ n4*100) / N, onde n1, n2, n3 e n4 = número de folíolos da amostra com as respectivas notas (1, 2, 3 e 4); $\mathrm{N}=$ total de folíolos da amostra.

A colheita foi feita de acordo com o ciclo das cultivares. Após a secagem natural das vagens em terreiro foram realizadas pesagens de produção em casca de cada subparcela, sendo os resultados originais submetidos a análise de variância.

Para as análises de variância de cada local, as avaliações de número de tripes/folíolo, notas de sintomas de tripes, notas de sintomas de verrugose foram transformados em $\sqrt{ } \mathrm{x}+0,5$ e os de índices de verrugose e de redução da produção em arco seno $\sqrt{x} / 100$, sendo as médias comparadas pelo teste de Tukey a $5 \%$ de probabilidade.

\section{RESULTADOS E DISCUSSÃO}

As avaliações do número de tripes por folíolo e das notas de sintomas apresentados nos folíolos, realizadas aos 56 DAP em Campinas e 57 DAP em Pindorama, quando ocorreu o pico da população da praga nos dois locais (Moraes et al., 2005), são apresentadas nas Tabelas 1 e 2, respectivamente.

O nível de infestação de tripes foi diferente nos dois locais, com Pindorama apresentando um nível de infestação inicial alto, por ser região produtora de amendoim com maior possibilidade de manter a população alta da praga. Verificouse também que as condições climáticas, principalmente em relação à quantidade e distribuição da precipitação, foram diferentes nos dois locais.

Em função dessas diferenças e da comparação das variâncias nos dois locais, particularmente para as notas de avaliação dos sintomas de tripes ter apresentado quadrados médios residuais 5,9 vezes maior para tratamentos e 6,4 vezes maior para cultivares, no experimento de Pindorama em relação ao de Campinas, os resultados foram analisados separadamente.

Os resultados mostraram a eficiência do inseticida thiamethoxam no controle do tripes, com diferenças significativas entre as médias dos tratamentos com e sem aplicação do inseticida, tanto para as avaliações do número de tripes por folíolo, como para os sintomas do tripes nos folíolos, nos dois locais (Tabelas 1 e 2). A eficiência desse inseticida já havia sido relatada no controle de pragas de outras culturas (Belletini et al., 2000; Benvenga et al., 1998; Raetano et al., 2000; Martins \& Nakamura, 2000; Senn et al., 2000a, b).

Segundo Belletini et al. (2000), o thiamethoxam usado em tratamento de sementes mostrou eficiente controle de tripes do algodoeiro, proporcionando o aumento na altura das plantas e na produção de sementes. Os efeitos do tripes e do seu controle sobre o desenvolvimento vegetativo e a produção de várias cultivares de amendoim, também foram observados por Moraes (2005).

As cultivares apresentaram diferenças significativas apenas para a média dos tratamentos na avaliação dos sintomas através de escala de notas em Campinas (Tabela 1), onde se observou uma redução dos sintomas variando entre 20 e 26\% nas parcelas onde o thiamethoxam foi aplicado. Já em Pindorama, a redução dos sintomas do tripes nas parcelas tratadas foi maior, variando entre 31 e $42 \%$ (Tabela 2). As cultivares de porte rasteiro, principalmente o Runner IAC 886 e Tégua, mostraram sintomas do ataque do tripes menos severos que as cultivares de porte ereto.

As diferenças entre as cultivares não foram significativas nas avaliações do número de tripes/folíolo nos dois locais (Tabelas 1 e 2), sendo observadas reduções na população do tripes nas parcelas tratadas com o thiamethoxam, variando entre 56 e 70\%, em Pindorama e entre 68 e $73 \%$, em Campinas.

A tendência das cultivares de porte rasteiro, particularmente do IAC-Caiapó, de serem menos atacados pelo tripes na ausência de controle químico, relatada por Gabriel et al. (1996, 1998) somente foi observada em relação as cultivares de porte rasteiro, apesar das análises de variância não indicarem diferenças significativas.

Apesar do bom controle das principais doenças foliares (manchas castanha e preta, ferrugem) através da utilização do difenoconazole, foi observado um controle apenas parcial da verrugose. Moraes et al. (2001) verificaram que o difenoconazole destacou-se entre outros fungicidas pela eficiência no controle da verrugose na cultivar Tatu, proporcionando uma redução média de $29 \%$ na intensidade da doença.

As avaliações da verrugose nas hastes, pecíolos e hastes e pecíolos, tanto através da escala de notas de sintomas como do índice da doença, mostraram que, quando foi aplicado o thiamethoxam para o controle do tripes, houve redução na severidade da verrugose nos dois locais (Tabela 3 e 4).

Nos dois locais as maiores severidades de verrugose foram observadas nas cultivares de porte ereto, com as três cultivares mostrando comportamento semelhante. Entre as cultivares rasteiros, o IAC-Caiapó apresentou os menores índices de severidade da verrugose na média das parcelas tratadas e não tratadas com o inseticida (Tabelas 3 e 4).

As maiores produções (em $\mathrm{kg} / \mathrm{ha}$ ) nos dois locais (Tabela 5), foram obtidas nas parcelas tratadas com o 
Efeito da aplicação de thiamethoxam para o controle de tripes...

TABELA 1 - Avaliações de número de tripes por folíolo e notas de sintomas nos folíolos, em seis cultivares de amendoim (Arachis hypogaea), aos 57 dias do plantio, nas parcelas tratadas (T) e não tratadas (NT) com inseticida, sob condições de infestação natural em campo. Pindorama, SP, 2001/2002

\begin{tabular}{|c|c|c|c|c|c|}
\hline \multirow{2}{*}{$\begin{array}{l}\text { Avaliações } \\
\text { de tripes }{ }^{1}\end{array}$} & \multirow{2}{*}{ Cultivares } & \multicolumn{2}{|c|}{ Tratamentos } & \multirow{2}{*}{$\begin{array}{l}\text { Médias de } \\
\text { Cultivares }^{3}\end{array}$} & \multirow{2}{*}{$\begin{array}{c}\text { \% de redução } \\
\text { T / NT }\end{array}$} \\
\hline & & Tratado & Não tratado & & \\
\hline \multirow{6}{*}{$\begin{array}{l}\text { Número de } \\
\text { tripes por } \\
\text { folíolo }\end{array}$} & IAC-Tatu-ST & 0,74 & 2,11 & 1,43 & 65 \\
\hline & IAC 5 & 0,90 & 2,52 & 1,71 & 64 \\
\hline & IAC 22 & 0,81 & 2,43 & 1,62 & 67 \\
\hline & Rumer IAC 886 & 0,77 & 2,57 & 1,67 & 70 \\
\hline & Tégua & 0,80 & 2,31 & 1,56 & 65 \\
\hline & IAC-Caiapó & 0,83 & 1,87 & 1,35 & 56 \\
\hline \multicolumn{2}{|c|}{ Média de tratamentos $^{2}$} & $0,81 \mathrm{~B}$ & $2,30 \mathrm{~A}$ & & \\
\hline & IAC-Tatu-ST & 1,32 & 1,97 & 1,65 & 33 \\
\hline Notas de & IAC 5 & 1,29 & 2,13 & 1,71 & 39 \\
\hline Sintomas & IAC 22 & 1,30 & 2,18 & 1,74 & 40 \\
\hline \multirow[t]{3}{*}{ de tripes } & Runner IA C 886 & 1,26 & 2,17 & 1,72 & 42 \\
\hline & Tégua & 1,24 & 1,94 & 1,59 & 36 \\
\hline & IAC-Caiapó & 1,29 & 1,87 & 1,58 & 31 \\
\hline \multicolumn{2}{|c|}{ Média de tratamentos $^{2}$} & $1,28 \mathrm{~B}$ & $2,04 \mathrm{~A}$ & & \\
\hline
\end{tabular}

TABELA 2 - Avaliações de número de tripes por folíolo e notas de sintomas nos folíolos, em seis cultivares de amendoim (Arachis hypogaea), aos 56 dias do plantio, nas parcelas tratadas (T) e não tratadas (NT) com inseticida, sob condições de infestação natural em campo. Campinas, SP, 2001/2002

\begin{tabular}{|c|c|c|c|c|c|}
\hline \multirow{2}{*}{$\begin{array}{l}\text { Avaliações } \\
\text { de tripes } 1\end{array}$} & \multirow{2}{*}{ Cultivares } & \multicolumn{2}{|c|}{ Iratamentos } & \multirow{2}{*}{$\begin{array}{l}\text { Médias de } \\
\text { Cultivares }{ }^{3}\end{array}$} & \multirow{2}{*}{$\begin{array}{c}\text { \% de redução } \\
\text { T / NT }\end{array}$} \\
\hline & & Tratado & Não tratado & & \\
\hline \multirow{6}{*}{$\begin{array}{l}\text { Número de } \\
\text { tripes por } \\
\text { folíolo }\end{array}$} & IAC-Tatu-ST & 0,73 & 2,28 & 1,50 & 68 \\
\hline & IAC 5 & 0,73 & 2,72 & 1,73 & 73 \\
\hline & $\mathrm{IAC} 22$ & 0,75 & 2,38 & 1,57 & 68 \\
\hline & Runner IAC 886 & 0,73 & 2,38 & 1,56 & 69 \\
\hline & Tégua & 0,74 & 2,39 & 1,56 & 69 \\
\hline & IAC'-C'aiapó & 0,74 & 2,29 & 1,52 & 68 \\
\hline \multicolumn{2}{|c|}{ Média de tratamentos ${ }^{2}$} & $0,74 \mathrm{~B}$ & $2,41 \mathrm{~A}$ & & \\
\hline \multirow{6}{*}{$\begin{array}{l}\text { Notas de } \\
\text { sintomas de } \\
\text { tripes }\end{array}$} & IAC-Tatu-ST & 1,42 & 1,81 & $1,62 \mathrm{a}$ & 22 \\
\hline & IAC 5 & 1,42 & 1,92 & 1,67 a & 26 \\
\hline & IAC 22 & 1,41 & 1,88 & $1,65 \mathrm{a}$ & 25 \\
\hline & Runner IAC 886 & 1,34 & 1,67 & $1,50 \mathrm{~b}$ & 20 \\
\hline & Tégua & 1,31 & 1,66 & $1,48 \mathrm{~b}$ & 21 \\
\hline & IAC-Caiapó & 1,39 & 1,77 & $1,58 \mathrm{ab}$ & 21 \\
\hline \multicolumn{2}{|c|}{ Média de tratamentos ${ }^{2}$} & $1,38 \mathrm{~B}$ & $1,78 \mathrm{~A}$ & & \\
\hline
\end{tabular}

inseticida, com o IAC-Caiapó apresentando produções superiores às demais cultivares. Considerando que houve um controle parcial da verrugose (índices de verrugose abaixo de 40\%) através das aplicações de difenoconazole, as reduções na produção podem ser atribuídas principalmente aos danos causados pelos tripes, nas parcelas sem controle com o inseticida, como cita Moraes et al. (2005).

A IAC-Caiapó, cultivar que tem como característica a resistência parcial às doenças foliares e entre elas a verrugose (Godoy et al., 1996; Moraes \& Godoy, 1997), também tem mostrado tendência a ser menos atacado pelo tripes em ausência de controle químico (Gabriel et al., 1996, 1998). Essas características podem explicar as menores respostas na redução produção das parcelas não tratadas em relação às tratadas com o thiamethoxam (Tabela 5), observadas tanto em Campinas (23,9 \%), como em Pindorama (29,8 \%). As 
TABELA 3 - Avaliações de verrugose em seis cultivares de amendoim (Arachis hypogaea), através de notas de sintomas (escala variando de 1 a 4) e índice (variando de 0 a 100\%) em hastes, pecíolos e hastes e pecíolos, aos 92 dias do plantio, em Pindorama, 2001/2002

\begin{tabular}{|c|c|c|c|c|c|c|c|}
\hline \multirow{2}{*}{ Amostra } & \multirow{2}{*}{ Cultivares } & \multicolumn{3}{|c|}{ Notas de sintomas da verrugose 1} & \multicolumn{3}{|c|}{ Índice de verrugose $(\%)^{2}$} \\
\hline & & Tratado & Não tratado ${ }^{4}$ & Média $^{5}$ & Tratado & Não tratado & Média $^{5}$ \\
\hline \multirow{6}{*}{ Haste } & IAC-Tatu-ST & 1,06 & 1,44 a & $1,25 \mathrm{a}$ & 8,47 & 31,62 & $20,04 \mathrm{ab}$ \\
\hline & IAC 5 & 1,04 & $1,42 \mathrm{a}$ & $1,23 \mathrm{ab}$ & 6,70 & 30,53 & $18,61 \mathrm{ab}$ \\
\hline & IAC 22 & 1,10 & $1,40 \mathrm{ab}$ & $1,25 \mathrm{a}$ & 12,18 & 29,4 & 20,79 a \\
\hline & Runner IAC 886 & 1,00 & $1,23 \mathrm{c}$ & $1,12 \mathrm{c}$ & 1,52 & 20,6 & $11,06 \mathrm{c}$ \\
\hline & Tégua & 1,03 & $1,25 \mathrm{bc}$ & $1,14 \mathrm{bc}$ & 6,06 & 21,99 & $14,03 \mathrm{bc}$ \\
\hline & & & & $1,10 \mathrm{c}$ & 0,00 & 19,67 & $9,83 \mathrm{c}$ \\
\hline \multirow{2}{*}{\multicolumn{2}{|c|}{$\begin{array}{l}\text { Média de tratamentos } \\
\text { CV \% }\end{array}$}} & $1,04 \mathrm{~B}$ & $1,33 \mathrm{~A}$ & & $5,82 \mathrm{~B}$ & $25,63 \mathrm{~A}$ & \\
\hline & & $3,92^{3}$ & & $7,38^{5}$ & $21,36^{3}$ & & $34,96^{5}$ \\
\hline \multirow{6}{*}{ Pecíolo } & IAC-Tatu-ST & 1,08 & 1,46 & $1,27 \mathrm{a}$ & 10,27 & 33,86 & 22,06 a \\
\hline & IAC 5 & 1,04 & 1,32 & $1,18 \mathrm{abc}$ & 4,59 & 25,42 & $15,00 \mathrm{bc}$ \\
\hline & IAC 22 & 1,10 & 1,42 & $1,26 \mathrm{ab}$ & 12,04 & 30,27 & $21,16 \mathrm{ab}$ \\
\hline & Runner IAC 886 & 1,00 & 1,30 & $1,15 \mathrm{bc}$ & 0,00 & 24,36 & $12,18 \mathrm{c}$ \\
\hline & Tégua & 1,00 & 1,27 & $1,14 \mathrm{c}$ & 0,00 & 23,05 & $11,52 \mathrm{c}$ \\
\hline & IAC-Caiapó & 1,00 & 1,21 & $1,10 \mathrm{c}$ & 0,00 & 19,67 & $9,83 \mathrm{c}$ \\
\hline \multirow{2}{*}{\multicolumn{2}{|c|}{$\begin{array}{l}\text { Média de tratamentos }{ }^{3} \\
\mathrm{CV} \%\end{array}$}} & $1,04 \mathrm{~B}$ & $1,33 \mathrm{~A}$ & & $4,48 \mathrm{~B}$ & $26,10 \mathrm{~A}$ & \\
\hline & & $3,07^{3}$ & & $7,67^{5}$ & $17,62^{3}$ & & $36,60^{5}$ \\
\hline \multirow{6}{*}{ Haste e Pecíolo } & IAC-Tatu-ST & 1,07 & 1,45 & $1,26 \mathrm{a}$ & 9,74 & 32,84 & $21,29 \mathrm{a}$ \\
\hline & IAC 5 & 1,04 & 1,37 & $1,21 \mathrm{ab}$ & 6,51 & 28,22 & $17,37 a b$ \\
\hline & IAC 22 & 1,10 & 1,41 & $1,26 \mathrm{a}$ & 12,89 & 29,92 & $21,40 \mathrm{a}$ \\
\hline & Runner IAC 886 & 1,00 & 1,27 & $1,14 \mathrm{bc}$ & 1,07 & 22,81 & $11,94 \mathrm{bc}$ \\
\hline & Tégua & 1,02 & 1,26 & $1,14 \mathrm{bc}$ & 4,28 & 22,65 & $13,47 \mathrm{bc}$ \\
\hline & IAC-Caiapó & 1,00 & 1,21 & $1,10 \mathrm{c}$ & 0,00 & 17,76 & $9,98 \mathrm{c}$ \\
\hline \multirow{2}{*}{\multicolumn{2}{|c|}{$\begin{array}{l}\text { Média de tratamentos }{ }^{3} \\
\mathrm{CV} \%\end{array}$}} & $1,04 \mathrm{~B}$ & $1,33 \mathrm{~A}$ & & $5,75 \mathrm{~B}$ & $26,03 \mathrm{~A}$ & \\
\hline & & $3,18^{3}$ & & $6,21^{5}$ & $16,26^{3}$ & & $28,86^{5}$ \\
\hline
\end{tabular}

tDados originais transformados em $\sqrt{ } \mathrm{x}+0,5$ para a análise de variância

${ }^{2}$ Dados originais transformados em arco seno $\sqrt{\mathrm{x}} / 100$ para a análise de variância.

${ }^{3}$ Médias de tratamentos seguidas por letras maiúscula distintas diferem entre si, pelo teste de Tukey a 5\% de probabilidade.

${ }^{4}$ Médias de verrugose em haste nos cultivares, dentro dos tratamentos, seguidas por letras minúsculas distintas diferem entre si, pelo teste de Tukey a 5\% de probabilidade.

${ }^{5}$ Médias de verrugose nos cultivares seguidas por letras minúsculas distintas diferem entre si, pelo teste de Tukey a 5\% de probabilidade.

cultivares Runner IAC 886 e Tégua foram as que mostraram os maiores índices de redução na produção nas parcelas não tratadas com o inseticida, nos dois locais.

A ação de inseticidas utilizados no controle de pragas sobre algumas doenças já havia sido verificada anteriormente. Richardson (1959) observou que aplicações de aldrin e endrin no solo reduziram a murcha do tomateiro, causada por Fusarium oxysporum f. sp. lycopersici e que aldrin, dieldrin e endrin diminuíram a incidência foliar de Alternaria solani Sorauer. O inseticida BHC (hexaclorociclobenzeno), empregado no tratamento de sementes de cereais tem mostrado eficiência no controle de Tilletia caries (DC.) Tul. \& C. Tul e T. controversa J.G. Kühn (Tyler \& Jansen, 1963; Purdy, 1965)

O inseticida cartap, utilizado para o controle de diversas espécies de insetos, mostrou efeito fungicida sobre Uromyces appendiculatus F. Strauss, causador da ferrugem do feijoeiro (Ito et al., 1995). Esse inseticida mostrou ação erradicante, inviabilizando os uredosporos, enquanto que os fungicidas normalmente empregados no controle dessa doença apresentam ação preventiva eficiente e pequena ação curativa, não apresentando efeito sobre uredosporos maduros.
Apesar de vários relatos da eficiência do thiamethoxam no controle de alguns insetos sugadores de importância econômica (Belletini et al., 2000; Benvenga et al., 1998; Raetano et al., 2000; Hofer et al., 2000; Martins \& Nakamura, 2000; Senn et al., 2000a), não existem constatações da sua ação sobre qualquer agente causal de doenças de plantas.

Os resultados obtidos mostraram claramente que, além da eficiência do thiamethoxam no controle do tripes do amendoim, esse inseticida proporcionou uma ação aditiva no controle da verrugose, numa interação de controle com o fungicida difenoconazole, como mostram os resultados, com a redução tanto do tripes como da verrugose nas parcelas tratadas com esse inseticida, nos dois locais.

A relação entre a eficiência do thiamethoxam no controle do tripes e a diminuição da severidade da verrugose não está bem esclarecida. No entanto, parece ser devida a algum mecanismo de ação diretamente sobre o fungo e não indireto no controle do tripes, visto que o fungo $S$. arachidis é capaz de penetrar diretamente pela cutícula, independentemente de ferimentos causados pelos insetos ou outros mecanismos de penetração (Moraes \& Godoy, 1987). Além disso, o acompanhamento de culturas de amendoim em diferentes anos e locais tem mostrado altas severidades 
Efeito da aplicação de thiamethoxam para o controle de tripes...

TABELA 4 - Avaliações de verrugose em seis cultivares de amendoim (Arachis hypogaea), através de notas de sintomas (escala variando de 1 a 4) e índice (variando de 0 a 100\%) em hastes, pecíolos e hastes e pecíolos, aos 79 dias do plantio, em Campinas, 2001/2002

\begin{tabular}{|c|c|c|c|c|c|c|c|}
\hline \multirow{2}{*}{ Amostra } & \multirow{2}{*}{ Cultivares } & \multicolumn{3}{|c|}{ Notas de sintomas da verrugose 1} & \multicolumn{3}{|c|}{ Índice de verrugose $(\%)^{2}$} \\
\hline & & Tratado & Não tratado & Média $^{4}$ & Tratado & Não tratado & Média $^{4}$ \\
\hline \multirow{6}{*}{ Haste } & IAC-Tatu-ST & 1,22 & 1,53 & $1,37 a b$ & 19,88 & 37,42 & $28,65 \mathrm{ab}$ \\
\hline & IAC 5 & 1,30 & 1,47 & $1,38 \mathrm{ab}$ & 24,88 & 32,78 & $28,83 \mathrm{ab}$ \\
\hline & IAC 22 & 1,38 & 1,55 & 1,46 a & 28,56 & 36,81 & $32,69 \mathrm{a}$ \\
\hline & Runner IAC 886 & 1,04 & 1,32 & $1,18 \mathrm{c}$ & 4,80 & 25,63 & $15,22 \mathrm{c}$ \\
\hline & Tégua & 1,13 & 1,34 & $1,24 \mathrm{bc}$ & 13,69 & 25,46 & $19,57 \mathrm{bc}$ \\
\hline & IAC-Caiapó & 1,00 & 1,22 & $1,11 \mathrm{c}$ & 0,00 & 19,70 & $9,85 \mathrm{c}$ \\
\hline \multirow{2}{*}{\multicolumn{2}{|c|}{$\begin{array}{l}\text { Média de tratamentos }{ }^{3} \\
\mathrm{CV} \%\end{array}$}} & $1,18 \mathrm{~B}$ & $1,40 \mathrm{~A}$ & & $15,30 \mathrm{~B}$ & $29,64 \mathrm{~A}$ & \\
\hline & & $4,20^{3}$ & & $11,00^{4}$ & $36,17^{3}$ & & $16,55^{4}$ \\
\hline \multirow{6}{*}{ Pecíolo } & IAC-Tatu-ST & 1,08 & 1,26 & $1,17 a b c$ & 10,27 & 21,91 & $16,09 \mathrm{ab}$ \\
\hline & IAC 5 & 1,10 & 1,26 & $1,18 \mathrm{ab}$ & 11,92 & 22,51 & $17,22 \mathrm{a}$ \\
\hline & IAC 22 & 1,13 & 1,29 & $1,21 \mathrm{a}$ & 13,68 & 23,31 & $18,50 \mathrm{a}$ \\
\hline & Runner IAC 886 & 1,00 & 1,08 & $1,04 \mathrm{c}$ & 0,00 & 8,30 & $4,15 \mathrm{c}$ \\
\hline & Tégua & 1,02 & 1,13 & $1,07 \mathrm{bc}$ & 2,15 & 13,32 & $7,74 \mathrm{bc}$ \\
\hline & IAC-Caiapó & 1,00 & 1,08 & $1,04 \mathrm{c}$ & 0,00 & 8,30 & $4,15 \mathrm{c}$ \\
\hline \multirow{2}{*}{\multicolumn{2}{|c|}{$\begin{array}{l}\text { Média de tratamentos }{ }^{3} \\
\mathrm{CV} \%\end{array}$}} & $1,05 \mathrm{~B}$ & $1,18 \mathrm{~A}$ & & $6,34 \mathrm{~B}$ & $16,28 \mathrm{~A}$ & \\
\hline & & $2,80^{3}$ & & $9,70^{4}$ & $17,66^{3}$ & & $68,12^{4}$ \\
\hline \multirow{6}{*}{ Haste e Pecíolo } & IAC-Tatu-ST & 1,15 & 1,40 & $1,28 \mathrm{ab}$ & 16,06 & 30,13 & $23,09 \mathrm{ab}$ \\
\hline & IAC 5 & 1,21 & 1,38 & $1,29 \mathrm{ab}$ & 19,72 & 28,06 & $23,89 \mathrm{a}$ \\
\hline & IAC 22 & 1,26 & 1,42 & $1,34 \mathrm{a}$ & 22,61 & 30,56 & $26,59 \mathrm{a}$ \\
\hline & Runner IAC 886 & 1,02 & 1,21 & $1,11 \mathrm{c}$ & 3,38 & 19,34 & $11,36 \mathrm{c}$ \\
\hline & Tégua & 1,08 & 1,24 & $1,16 b c$ & 9,94 & 20,55 & $15,24 \mathrm{bc}$ \\
\hline & IAC-Caiapó & 1,00 & 1,16 & $1,08 \mathrm{c}$ & 0,00 & 15,63 & $7,82 \mathrm{c}$ \\
\hline \multirow{2}{*}{\multicolumn{2}{|c|}{$\begin{array}{l}\text { Média de tratamentos }{ }^{3} \\
\mathrm{CV} \%\end{array}$}} & $1,12 \mathrm{~B}$ & $1,30 \mathrm{~A}$ & & $11,95 \mathrm{~B}$ & $24,05 \mathrm{~A}$ & \\
\hline & & $3,39^{3}$ & & $9,87^{4}$ & $16,49^{3}$ & & $38,21^{4}$ \\
\hline
\end{tabular}

${ }^{1}$ Dados originais transformados em $\sqrt{ } \mathrm{x}+0,5$ para a análise de variância;

${ }^{2}$ Dados originais transformados em arco seno $\sqrt{\mathrm{x}} / 100$ para a análise de variância;

${ }^{3}$ Médias de tratamentos seguidas por letras maiúsculas distintas diferem entre si, pelo teste de Tukey a 5\% de probabilidade;

${ }^{4}$ Médias de verrugose nos cultivares seguidas por letras minúsculas distintas diferem entre si, pelo teste de Tukey a 5\% de probabilidade.

TABELA 5 - Produção total (kg/ha) e índice de redução na produção (\%) em seis cultivares de amendoim (Arachis hypogaea), em parcelas tratadas e não tratadas com inseticidas, em condições de campo, em Pindorama e Campinas, 2001/2002

\begin{tabular}{|c|c|c|c|c|}
\hline \multirow{2}{*}{ Local } & \multirow{2}{*}{ Cultivares } & \multicolumn{2}{|c|}{ Produção em kg/ha } & \multirow{2}{*}{$\begin{array}{l}\text { Índice de } \\
\text { redução }(\%)^{3}\end{array}$} \\
\hline & & Tratado $^{2}$ & Não tratado ${ }^{2}$ & \\
\hline \multirow{6}{*}{ Pindorama } & IAC-Tatu-ST & $2685 \mathrm{c}$ & $1943 \mathrm{~b}$ & $31,7 \mathrm{~cd}$ \\
\hline & IAC 5 & $3313 \mathrm{ab}$ & $1757 \mathrm{~b}$ & $42,9 \mathrm{abc}$ \\
\hline & IAC 22 & $3116 \mathrm{bc}$ & 1944 b & 36,8 bcd \\
\hline & Runner IAC 886 & $3625 \mathrm{ab}$ & $1392 \mathrm{~b}$ & $51,3 \mathrm{a}$ \\
\hline & Tégua & $3182 \mathrm{abc}$ & $1400 \mathrm{~b}$ & $48,1 \mathrm{ab}$ \\
\hline & IAC-Caiapó & 3796 a & 2811 a & $29,8 \mathrm{~d}$ \\
\hline \multirow{2}{*}{\multicolumn{2}{|c|}{ Média de tratamentos ${ }^{1}$}} & $3286 \mathrm{~A}$ & $1874 \mathrm{~B}$ & \\
\hline & & $9,36^{1}$ & & $16,46^{3}$ \\
\hline \multirow{6}{*}{ Campinas } & IAC-Tatu-ST & 3895 & 2911 & $24,5 \mathrm{ab}$ \\
\hline & IAC 5 & 3681 & 2795 & $27,5 \mathrm{abc}$ \\
\hline & IAC 22 & 3972 & 2964 & $27,5 \mathrm{ab}$ \\
\hline & Runner IAC 886 & 3986 & 2830 & $32,2 \mathrm{ab}$ \\
\hline & Tégua & 4010 & 2382 & $39,5 \mathrm{a}$ \\
\hline & IAC-Caiapó & 4130 & 3332 & $23,9 \mathrm{~b}$ \\
\hline \multirow{2}{*}{\multicolumn{2}{|c|}{$\begin{array}{l}\text { Média de tratamentos }{ }^{1} \\
\mathrm{CV} \%\end{array}$}} & $3946 \mathrm{~A}$ & $2869 \mathrm{~B}$ & \\
\hline & & $5,12^{1}$ & & $28,52^{3}$ \\
\hline \multicolumn{5}{|c|}{$\begin{array}{l}\text { Médias de tratamentos seguidas por letras maiúscula distintas diferem } \\
\text { entre si, pelo teste de Tukey a } 5 \% \text { de probabilidade. }\end{array}$} \\
\hline \multicolumn{5}{|c|}{$\begin{array}{l}{ }^{2} \text { Médias de produção dos cultivares em Pindorama, dentro dos tratamentos, } \\
\text { seguidas por letras minúsculas distintas diferem entre si, teste Tukey a } \\
5 \% \text { de probabilidade. }\end{array}$} \\
\hline \multicolumn{5}{|c|}{$\begin{array}{l}{ }^{3} \text { Porcentagens médias de redução na produção dos cultivares transformadas em } \\
\text { arco seno } \sqrt{x}_{\mathrm{x}} / 100 \text { para a análise de variância, seguidas por letras minúsculas } \\
\text { distintas diferem entre si, pelo teste Tukey a } 5 \% \text { de probabilidade. }\end{array}$} \\
\hline
\end{tabular}

da verrugose, mesmo com baixos níveis de infestação de tripes, ou presença não significativa da verrugose mesmo com infestações elevadas do tripes.

Os resultados obtidos têm grande importância para o manejo integrado de pragas e doenças, prática que vem sendo enfatizada para aumentar a produtividade com custos menores e menores prejuízos ao ambiente. A ação do thiamethoxam controlando uma das principais pragas do amendoim e reduzindo a severidade da verrugose deve ser melhor explorada.

\section{REFERÊNCIAS BIBLIOGRÁFICAS}

BARRETO, M. Doenças do amendoim (Arachis hypogaea L.). In: Kimati, H., Amorim, L., Bergamin Filho, A., Camargo, I.E.A. \& Rezende, J.A.M. (Eds.) Manual de Fitopatologia. v. 2. Doenças das Plantas Cultivadas. 3a. ed., São Paulo SP. Ed. Agronômica Ceres Ltda. 1997. pp. 63-77.

BATISTA, G. C., GALLO D. \& CARVALHO, R.P.L. Determinação do período crítico de ataque do tripes do amendoim, Enneothrips flavens Moulton, 1941, em cultura “das águas”. Anais da Sociedade Entomológica do Brasil 2:45-53. 1973.

BELlETINI, S., ARAMAKI, P.H., BIAGGI, L.S., MINUCCI, A. \& SILVA, W.G. Effect of different seed treatments on trips Frankiniella schultzei control - vegetative development and 
yield of cotton crop. Abstracts, XXI International Congress of Entomology. Foz do Iguaçu PR. 2000. p. 49.

BENVENGA, S.R., SILVA, J.L., PAIVA, P.E.B., GRAVENA, R. \& GRAVENA, S. Inseticidas mais eficientes no controle das cigarrinhas Acrogonia racilis, Dilobopterus costalimai e Oncometopia facialis em citros. Resumos, XVI Congresso Brasileiro de Entomologia. Rio de Janeiro RJ. 1998. p. 372.

BITANCOURT, A.A. \& JENKINS, A.E. Novas espécies de Elsinoe e Sphaceloma sobre hóspedes de importância econômica. Archivos Instituto Biológico 11:45-58. 1940.

CALCAGNOLO, G. \& LEITE, F.M. \& GALLO, J.R. Efeitos da infestação do tripes nos folíolos do amendoinzeiro Enneothrips (Enneothripiella) flavens Moulton, 1941, no desenvolvimento das plantas, na qualidade da produção de uma cultura “das águas”. O Biológico 40:241-242. 1974.

DARIO, G.J.A., LEITE, O.M.C. \& DARIO, P.W. Avaliação da eficiência do difenoconazole no controle de fungos que atacam a parte aérea do amendoim. Fitopatologia Brasileira 19 (Supl.):283. 1994.

GABRIEL, D., NOVO, J.P.S., GODOY, I.J. \& BARBOZA, J.P. Flutuação populacional de Enneothrips flavens Moulton em cultivares de amendoim. Bragantia 55:253-257. 1996.

GABRIEL, D., NOVO, J.P.S. \& GODOY, I.J. Efeito do controle químico na população de Enneothrips flavens Moulton e na produtividade de cultivares de amendoim Arachis hypogaea L. O Biológico 65:51-56. 1998.

GALLO, D., NAKANO, O., SILVEIRA NETO, S., CARVALHO, R.P.L., BATISTA, G.C., BERTI FILHO, E., PARRA, J.R.P., ZUCCHI, R.A., ALVES, S.B., VENDRAMIN, J.D., MARCHINI, L.C., LOPES, J.R.S. \& OMOTO, C. Entomologia Agrícola. Piracicaba SP. FEALQ. 2002.

GODOY, I.J., MORAES, S.A., TURATTI, J.M., PEREIRA, J.C.V.N.A., MARTINS, A.L.M. \& PAULO, E.M. IAC-Caiapó: novo cultivar de amendoim. Instituto Agronômico de Campinas. Folder. 4 p. 1996.

HOFER, D., BRANDL, F., ZANG, L. \& FOUGEROUX, A. Thiamethoxam (Cruiser) as seed treatment - value beyond insect control. Abstracts, XXI. International Congress of Entomology. Foz do Iguaçu PR. 2000. p. 337.

IAC - INSTITUTO AGRONÔMICO. Cultivar de amendoim IACTatu-ST. Instituto Agronômico de Campinas. Folder. 4p. 2000.

IAC - INSTITUTO AGRONÔMICO. Cultivares de amendoim: novas opções para o mercado de confeitaria. Instituto Agronômico de Campinas. Folder. 12p. 2003.

ITO, M.F., BERGAMIN FILHO, A. \& CASTRO, J.L. Ação do inseticida cartap sobre a ferrugem do feijoeiro em campo. Fitopatologia Brasileira 20:577-584. 1995.

ITO, M.F., YUKI, V.A., DUDIENAS, C. \& TANAKA, M.A.S. Controle da ferrugem do feijoeiro através do inseticida cartap, em condições de laboratório. Anais do Seminário sobre pragas e doenças do feijoeiro, 4, Campinas. 1991. p. 20.

KOKALIS-BURELLE， N., PORTER, D.M., RODRÍGUEZKÁBANA, R., SMITH, D.H. \& SUBRAHMANYAM, P. (Eds.)
Compendium of peanut diseases. $2^{\text {nd }}$ ed., St. Paul MN. The American Phytopathological Society. 1997.

LASCA, D.H.C., GODOY, I.J., MARIOTTO, P.R., MORAES, S.A., JOCYS, T., ROSTON, A.J., PRATES, H.S. \& PELEGRINETTI, J.R. Controle de pragas e doenças da cultura do amendoim. Boletim Técnico CATI, Campinas, n. 174. 1983. p.10.

LEITE, O.M.C., De VICENZO, M.C.V. \& BALTIERI, E.M. Avaliação da eficiência do difenoconazole no controle de fungos que atacam a parte aérea do amendoim. Fitopatologia Brasileira 19:274. 1994. (Resumo)

MARTINS, J.C. \& NAKAMURA, G. Efficiency of seed treatments with thiamethoxam to control Bemisia argentifolii on cotton crop. Abstracts, XXI. International Congress of Entomology. Foz do Iguaçu PR. 2000. p. 343.

MORAES, A.R.A. Efeito da infestação de Enneothrips flavens Moulton no desenvolvimento e produtividade de seis cultivares de amendoim, em condições de campo (Dissertação de Mestrado). Instituto Agronômico de Campinas. 2005.

MORAES, A.R.A., LOURENÇÃO, A.L., GODOY, I.J. \& TEIXEIRA, G.C. Infestation by Enneothrips flavens Moulton and yield of peanut cultivars. Scientia Agricola 62:469-472. 2005.

MORAES, S.A. \& GODOY, I.J. Amendoim - Controle de Doenças. In: Vale, F.X.R. \& Zambolim, L. (Eds.) Controle de doenças de plantas. Grandes culturas. Viçosa MG. UFV - MAPA. Suprema Gráfica e Editora Ltda. v.1. 1997. pp. 1-49.

MORAES, S.A., GODOY, I.J., PEZZOPANE, J.R.M., PEREIRA, J.C.V.N.A. \& SILVEIRA, L.C.P. Eficiência de fungicidas no controle da mancha preta e verrugose do amendoim por método de monitoramento. Fitopatologia Brasileira 26:134-140. 2001.

PURDY, L.H. Common and dwarf bunts, their chemical control in Pacific Northwest. Plant Disease Reporter 49:42-46. 1965.

RAETANO, C.G., KUWAHARA, W.R., VINCH, R.R. \& KOBAYASHI, M.R. Influence of different application methods and dosage of thiamethoxam in thrips control. Abstracts, XXI. International Congress of Entomology. Foz do Iguaçu PR. 2000. p. 82.

RICHARDSON, L.T. Effect of inseticides and herbicides applied to soil on development of plant diseases. II. Early blight and Fusarium wilt of tomato. Canadian Journal of Plant Science 39:30-38. 1959.

ROSSETTO, C.J., RIBEIRO, I.J.A., POMPEU, A.S. \& IGUE, T. Interação entre tripes, a verrugose e variedades de amendoinzeiro. Ciência e Cultura 20:255. 1968.

SENN, R., HOFER, D., BRANDL, L. \& MORCOS, A. Thiamethoxam used as seed treatment (Cruiser/Adage) or as soil application (Actara/Platinum). Abstracts, XXI. International Congress of Entomology. Foz do Iguaçu PR. 2000a. p. 86.

SENN, R., FISHER, W., WYSS, P. \& MORCOS, A. Thiamethoxam: new innovative application opportunities for a systemic product. Abstracts, XXI. International Congress of Entomology. Foz do Iguaçu PR. 2000b. p. 320.

TYLER, L.J. \& JANSEN, N.F. Soil application of hexachlorobenzeno for control of dwarf bunt. Plant Disease Reporter 47:197-199. 1963. 